\title{
Dy uniform film morphologies on graphene studied with SPA-LEED and STM
}

\author{
D. McDougall a, b, H. Hattab a, M.T. Hershberger ${ }^{\text {a, b }}$, M. Hupalo a , M. Horn von Hoegen ${ }^{\text {d }}$, \\ P.A. Thiel ${ }^{\text {a, c }}$, M.C. Tringides ${ }^{\text {a, b, * }}$ \\ a Ames Laboratory, U.S. Department of Energy, Iowa State University, Ames, IA, 50011, USA \\ ${ }^{\mathrm{b}}$ Department of Physics and Astronomy, Iowa State University, Ames, IA, 50011, USA \\ ${ }^{\mathrm{c}}$ Department of Chemistry, Iowa State University, Ames, IA, 50011, USA \\ d Department of Physics and Center for Nanointegration CENIDE, University of Duisburg-Essen, Lotharstrasse 1, 47057, Duisburg, Germany
}

\section{A R T I C L E I N F O}

\section{Article history:}

Received 4 May 2016

Received in revised form

21 June 2016

Accepted 22 June 2016

Available online 1 July 2016

\begin{abstract}
A B S T R A C T
The use of graphene for microelectronics and spintronic applications requires strategies for metals to wet graphene and to grow layer-by-layer. This is especially important when metals will be used as electrical contacts or as spin filters. Extensive work in the literature so far has shown that this is very challenging, since practically all metals grow 3D, with multi-height islands forming easily. Reasons for the 3D morphology are the much weaker metal carbon bond when compared to the metal cohesive energy and the role of Coulomb repulsion of the poorly screened charges at the metal graphene interface. We employed the complementary techniques of SPA-LEED and STM to study the growth of Dy on graphene. It was found that under kinetic limitations it is possible to fully cover graphene with a bilayer Dy film, by growing well below room temperature in stepwise deposition experiments. The Dy film, however, is amorphous but ways to crystallize it within the 2D morphology are possible, since long range order improves at higher growth temperature.
\end{abstract}

Published by Elsevier Ltd.

\section{Introduction}

Understanding the growth of metals on graphene is essential for many future graphene applications. Metals are used as contacts for graphene-based devices so it is important to grow uniform metalgraphene interfaces with low contact resistance [1-6]. This requires finding conditions for the metals to wet and grow with uniform thickness, so the grown film is atomically flat and electron transport across the interface is coherent. A recent experimental study [1] has focused on identifying metal properties, which minimize contact resistance, expecting metals with the larger work function difference $\Delta \varphi$ with graphene to be the optimal choice. Surprisingly no such correlation was found between $\Delta \varphi$ and contact resistance; in addition for all deposited metals growth was 3D, irrespectively of the variation of $\Delta \varphi$. This shows that to control the contact quality, finding ways to tune the growth mode is essential. Understanding the role of thermodynamic factors to determine the

\footnotetext{
* Corresponding author. Ames Laboratory, U.S. Department of Energy, Iowa State University, Ames, IA, 50011, USA

E-mail address: mctringi@iastate.edu (M.C. Tringides).
}

growth mode and/or finding novel kinetic pathways are necessary. In a different application [7] graphene has been also proposed as a spin filter when sandwiched between magnetic metals (i.e., Ni and Co that have almost the same lattice constant as graphene). However this requires the grown morphology to be of uniform thickness so spin scattering is suppressed and only spins of the same polarization are transferred across graphene.

Recent STM experiments were carried out in parallel with theoretical studies using DFT to identify how the metal-carbon interaction determines the grown morphologies [8-12]. Graphene was prepared by the thermal annealing of SiC described in Ref. [8] of very high uniformity, as seen from the $\mu \mathrm{m}$-size domains of a single thickness. Standard tests were applied to distinguish single from bilayer graphene, i.e., the voltage dependence of the $6 \times 6$ and step height differences (with $0.33 \mathrm{~nm}$ the graphene step height indicating change of thickness; with $0.25 \mathrm{~nm}$ the SiC step height indicating thickness uniformity).

Practically all metals deposited (Pb, In, Mg, Fe, Eu, Dy, Gd), irrespectively of the strength of the metal-carbon interaction, were found to grow 3D (except possibly Eu which tends to show more extended islands, but with still a growth front with several 
incomplete layers exposed). The growth was found to be 3D even for very small deposited amount $\theta \sim 0.02 \mathrm{ML}$, very different from 2D growth, commonly seen at the onset of nucleation on metallic substrates [13]. 3D growth is also seen for metal growth on other types of graphene, i.e., exfoliated type [14]. This almost universal behavior is partially due to thermodynamics: the metal adsorption energy on graphene $\mathrm{E}_{\mathrm{a}}$ for practically all metals is much lower than the metal cohesive energy $E_{c}$, so for growth at room or higher temperature $3 \mathrm{D}$ morphology is favored. The propensity of metals not to wet graphene was seen in experiments where graphene (used as coating) was shown to reduce the wetting properties of the coated substrate [15].

Routes to grow 2D morphologies by manipulating growth can rely on kinetics, as already shown in several epitaxial systems $\sim 20$ years ago, when there was broad interest to study metal/metal and metal/semiconductor systems [16]. Such kinetic routes require lower temperatures and/or high flux rates and can be also applied to metal growth on graphene. Growth under these conditions results initially in high density of smaller islands which promote interlayer diffusion, thus completing a layer before the next layer nucleates on top. 2D growth on graphene was shown in Co films on graphene on $\operatorname{Ir}(111)$ [17] with Laser Pulse Deposition (LPD) with effective flux rates $\sim 10^{6}$ times higher than rates in typical epitaxial experiments. Introducing stress in thin films in controlled ways as a function of thickness can also result in modifying nucleation, it can increase island density and promote layer-by-layer growth [18].

In the current work we studied Dy growth on epitaxial graphene on SiC using two complementary techniques, Spot Profile Analysis Low Energy Electron Diffraction (SPA-LEED) and Scanning Tunneling Microscopy (STM). The use of SPA-LEED complements STM by monitoring growth in reciprocal space to present statistical information about the growth. Several spots can be measured simultaneously which reveal the statistical distribution of different surface phases (i.e, pristine graphene, graphene covered with Dy, buffer layer etc). These exploratory experiments carried over a wide temperature range $110 \mathrm{~K}-650 \mathrm{~K}$ confirm the growth of perfect Dy fcc(111) crystalline islands at $650 \mathrm{~K}$. More importantly they show that $2 \mathrm{D}$ growth is possible at lower temperature $\sim 110 \mathrm{~K}$ with more than $90 \%$ of the initial graphene substrate covered by depositing 2 ML. However the film is amorphous so it is important to find ways to crystallize it so its conductivity can become comparable to the metallic conductivity of crystalline Dy. Similar concern applies to the Co films grown with LPD and the similar requirement to find easy ways to crystallize them.

\section{Experiment}

SPA-LEED provides a representative and statistical picture of the growth (i.e. averaging over $10^{6}$ regions of coherent scattering on the surface). By studying the spot intensity distribution as a function of the normal and parallel momentum transfer components we can deduce detailed information about the island height, size, shape, etc. Variable temperature STM provides local information and directly confirms the structures from real space images.

The experiments were carried out in two separate UHV chambers one housing SPA-LEED and the other chamber an Omicron variable temperature STM. The flux rate in the STM experiments was determined by calculating the volume enclosed by the Dy film, since both the height and lateral extent are known from the piezo calibration. Calibration of the flux rate in the LEED chamber was based on stepwise deposition experiments until the graphene spot intensity drops to its minimum indicating a completely covered substrate. Since the growth temperature and morphology is similar in the SPA-LEED and STM experiments, we use the average film height of 2-layers (when the substrate is covered) for the SPA-LEED flux calibration. The temperatures were measured with Re-W (3\%$25 \%$ ) thermocouple spot-welded on the Ta clips holding the crystal in the LEED chamber and with a Si diode attached to the STM stage in the STM chamber.

The substrates used in the current experiments were 4- $\mathrm{H}$ and $6 \mathrm{H}-\mathrm{SiC}(0001)$ purchased from Cree, Inc. The samples were graphitized in UHV $\left(\mathrm{P} \sim 1 \times 10^{-10} \mathrm{mbar}\right)$ by direct current heating of the sample to $\sim 1500 \mathrm{~K}$ measured with an infrared pyrometer, with emissivity adjusted to $\varepsilon=0.85$, calibrated ex situ by comparing with a thermocouple attached to the sample. The layer thickness (whether single layer G1 or bilayer G2) was controlled by the heating rate: faster one-step heating rates (within $2-3 \mathrm{~s}$ to reach $1500 \mathrm{~K}$ ) result in large G1 domains while multiple heating steps with a slower rate $(30 \mathrm{~s}$ to reach $1500 \mathrm{~K}$ ) result in samples with large G2 areas. Dy growth and annealing was performed in a separate sample stage outside the STM head. Temperature from RT up to $850 \mathrm{~K}$ was reached by sample indirect heating and for a given power the temperature was measured by means of chromel-alumel thermocouple spot-welded to the heater. Higher temperatures to remove Dy films by desorption were reached through short flash annealing to $1400 \mathrm{~K}$.

\section{Experimental results}

Fig. 1 shows 1 -d scan through the specular spot along the [12 10] direction $(\mathrm{SiC}(\overline{10}))$ to $\mathrm{SiC}(10))$ for clean graphene. The spot shows the characteristic $6 \times 6$ satellite spots surrounding the 00 spot. The momentum transfer calibration was based on the $16.67 \% \mathrm{BZ}(100 \%$ $\mathrm{BZ}=$ size of Brillouin zone) position of the satellite spots. The 00 spot has a very unusual but highly reproducible shape, unique to

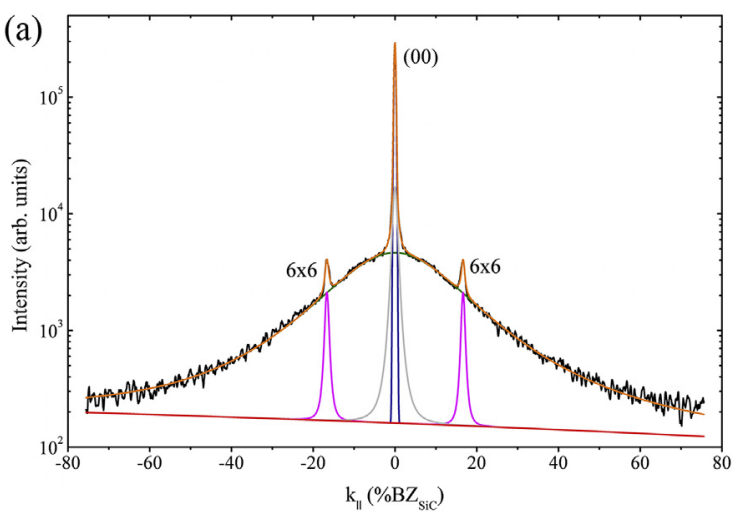

(b)

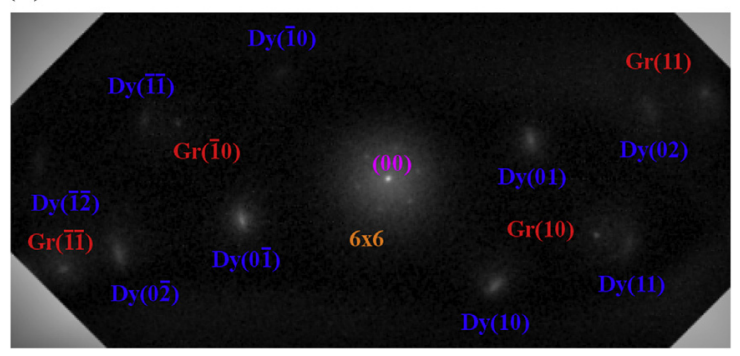

Fig. 1. (a) 1-d scan through the specular 00 spot at $E=142 \mathrm{eV}$ along the [1 $\overline{2} 10] \mathrm{di}-$ rection for clean graphene. The spot shows the characteristic $6 \times 6$ satellite spots surrounding the 00 spot. The fits were used to deduce the ratio of the central spike to the total integrated intensity. It was used as a measure of the quality of the initial surface. (b) 2-d pattern at E $=142 \mathrm{eV}$ and $2 \mathrm{ML}$ Dy deposited at $660 \mathrm{~K}$ shown over the accessible region of the Brillouin zone with all relevant spots marked. The two scanned directions are [12 10] (from $G(\overline{11})$ to $\operatorname{Gr}(11)$ ) and [1 $\overline{1} 00]$ from $(G(\overline{1} 0)$ ) to $G(10)$ ). (A colour version of this figure can be viewed online.) 
graphene, consisting of very sharp spike and a very broad (FWHM 50\%BZ) bell-shaped background. This background paradoxically indicates the formation of a single layer graphene [19] after it fully covers the buffer (zero) layer. Detailed experiments carried out as a function of energy both for the 00 and the graphene spot rule out that surface morphology (the oscillatory behavior of the two components as a function of scattering phase $\left.S=k_{z} /(2 \pi / d)\right)$ is the reason of the background. This unusual background is unique to graphene and most likely related to electron confinement in a truly ideal single layer coherent film [19]. Profile fitting of the full scan using a linear background over the full range, the broad 00 bellshaped background, the central spike and the two satellite $6 \times 6$ spots was applied to the profiles. The ratio of the spike to the broad component integrated intensities $\sim 0.8$ was a marker to ensure the same starting surface used for Dy deposition experiments.

Fig. 1(b) shows a 2-d pattern at $\mathrm{E}=142 \mathrm{eV}$ and $2 \mathrm{ML}$ Dy deposited at $660 \mathrm{~K}$ shown over the accessible region of the Brillouin

(a)

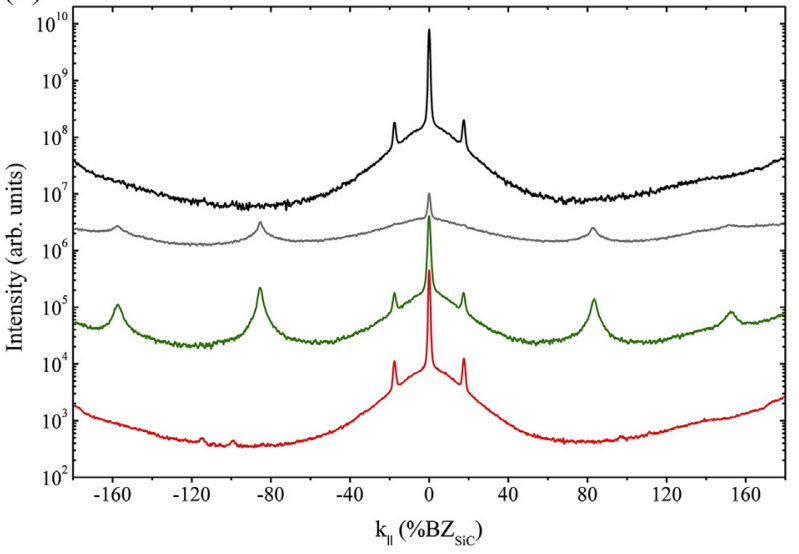

(b)
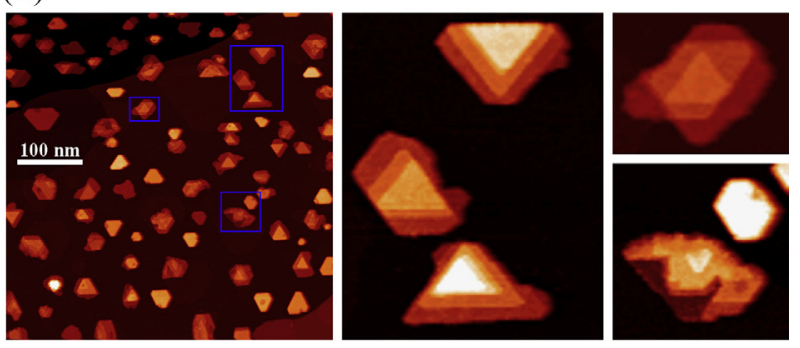

(c)

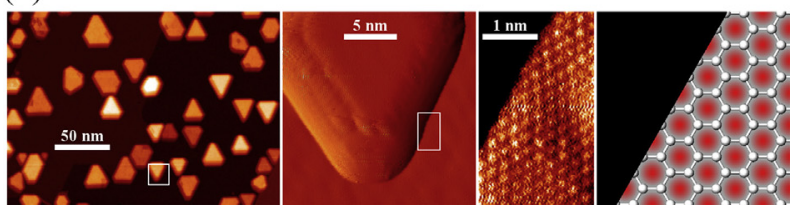

Fig. 2. (a) 1-d profiles at electron energy $E=142 \mathrm{eV}$ along [12 10]. The four profiles (from top to bottom) are for the initial surface, after $2 \mathrm{ML}$ Dy deposition at $650 \mathrm{~K}$ (collected at $650 \mathrm{~K}$ ), after cooling to $130 \mathrm{~K}$ and after heating to $1400 \mathrm{~K}$ to desorb Dy. A peak develops at the expected fcc(111) Dy position $86 \% \mathrm{BZ}$ (the ratio of the SiC to Dy lattice constants $0.307 / 0.358$ ). (b) STM $500 \times 500 \mathrm{~nm}^{2}$ image obtained after growth of 1.3 ML Dy at $650 \mathrm{~K}$ with the characteristic triangular island shapes of fcc(111) Dy. A large fraction of islands have incomplete tops with successive layers exposed, which generates the large wings of the Dy spot seen in (a). (c) The STM was used to confirm the Dy orientation along the [1100] direction as in Fig. 2(a). The insets show the graphene area zoomed successively to smaller scale indicating that the island edge is oriented 90 from the graphene lattice direction (the figure to the right is a schematic for comparison with the experimental images). (A colour version of this figure can be viewed online.) zone with all relevant spots marked. The two scanned directions are [12 10] (from $G(\overline{11})$ to $\operatorname{Gr}(11)$ ) and [1 $\overline{100}$ from $(G(\overline{10}))$ to $\mathrm{G}(10))$.

Fig. 2(a) shows 1 -d profiles at an electron energy $E=142 \mathrm{eV}$ along [1210] after deposition of Dy at higher temperature $\mathrm{T}=650 \mathrm{~K}$. It is known from earlier STM work that at this temperature crystalline multilayer Dy islands form [20]. This comparison can be used to cross-correlate the temperatures of the two experiments. The peaks at $86 \% \mathrm{BZ}$ are consistent with the expected position of the first integer order spots from fcc(111) Dy and reflects the epitaxial formation of Dy islands. The four profiles starting from the top are of the initial surface, after 2 ML Dy deposition at $650 \mathrm{~K}$ (collected also at $650 \mathrm{~K}$ ), after the surface was cooled to $130 \mathrm{~K}$ and the bottom profile after heating the surface to $1400 \mathrm{~K}$ to desorb Dy, respectively. The Dy spots taken at $650 \mathrm{~K}$ are weak because of the Debye-Waller factor; after cooling to $130 \mathrm{~K}$ the peak increases by a factor of $\sim 10$.

Compared with the graphene spots the Dy peaks are much broader. Under growth condition at $650 \mathrm{~K}$ after $2 \mathrm{ML}$ is deposited multi-height, 3D islands of average height $\sim 10$ layers and average width $\sim 15 \mathrm{~nm}$ form [21]. The islands have a range of heights (from 8 to 12 layers) and widths (from $10 \mathrm{~nm}$ to $20 \mathrm{~nm}$ ) which already causes significant finite size broadening of the Dy spots. In addition further spot broadening is related to the incomplete structure of individual islands. Fig. 2(b) shows an STM image obtained under similar conditions as Fig. 2(a) with the characteristic triangular island shapes of the fcc(111) stacking. The image also includes a large fraction of islands with incomplete tops with a few successive layers exposed. Since the phase of the scattered electron wave varies widely across the layers, destructive interference will be present within individual islands, which amounts to the island area being effectively smaller than its nominal area and can generate a much broader spot in Fig. 2(a).

Fig. 2(c) shows a different experiment with triangular Dy islands grown after a single $1.35 \mathrm{ML}$ deposition at $660 \mathrm{~K}$. The insets show the graphene area zoomed successively to smaller scale to determine the island orientation with respect to graphene. The black region in the third image is a Dy island and the surrounding area shows the graphene lattice atomically resolved, indicating that the

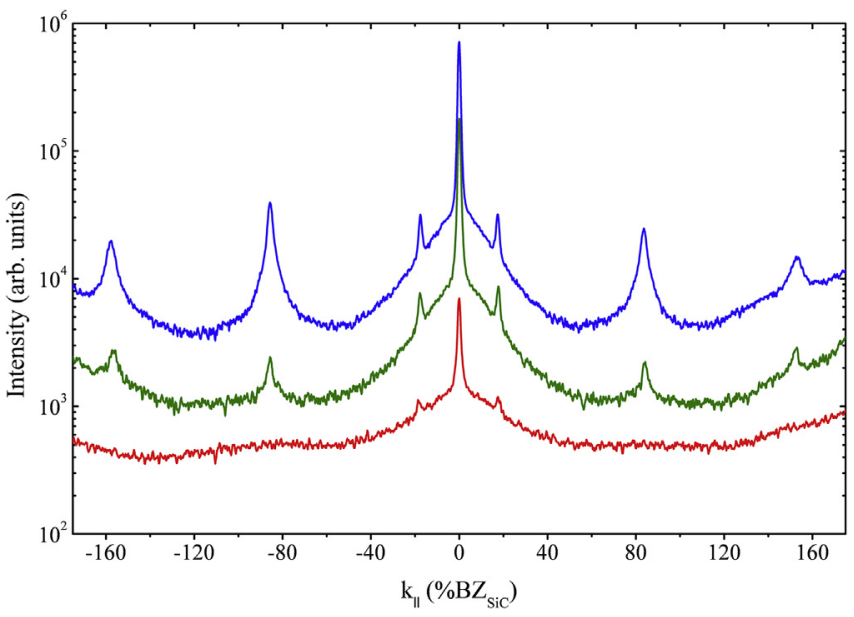

Fig. 3. LEED 1d-intensity profiles after deposition of $2 \mathrm{ML}, \mathrm{E}=142 \mathrm{eV}$ for different temperatures ( $650 \mathrm{~K}, \mathrm{RT}, 130 \mathrm{~K}$ ) along the [12 10] direction collected at $130 \mathrm{~K}$. No Dy spot is seen at $130 \mathrm{~K}$ and stronger Dy spots are seen with temperature indicating the gradual change from amorphous to crystalline Dy film. The integrated intensity of the Dy spot measures the fraction of the crystalline part of the film and the decrease of the $6 \times 6$ spot intensity the area covered by the film. (A colour version of this figure can be viewed online.) 
(a)

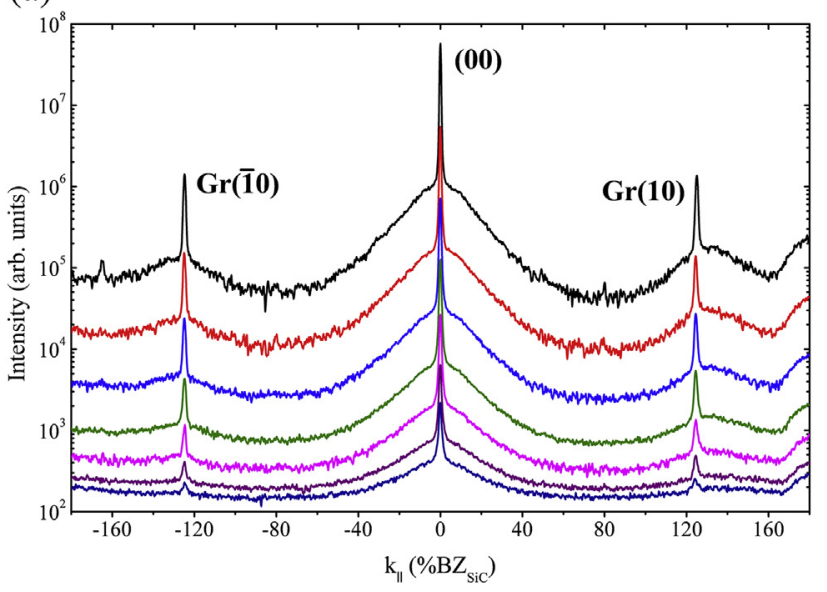

(b)

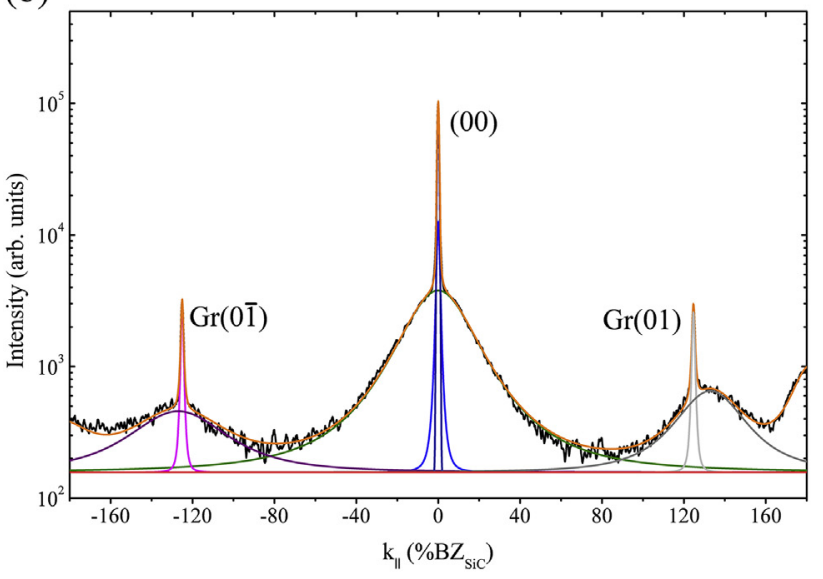

(c)

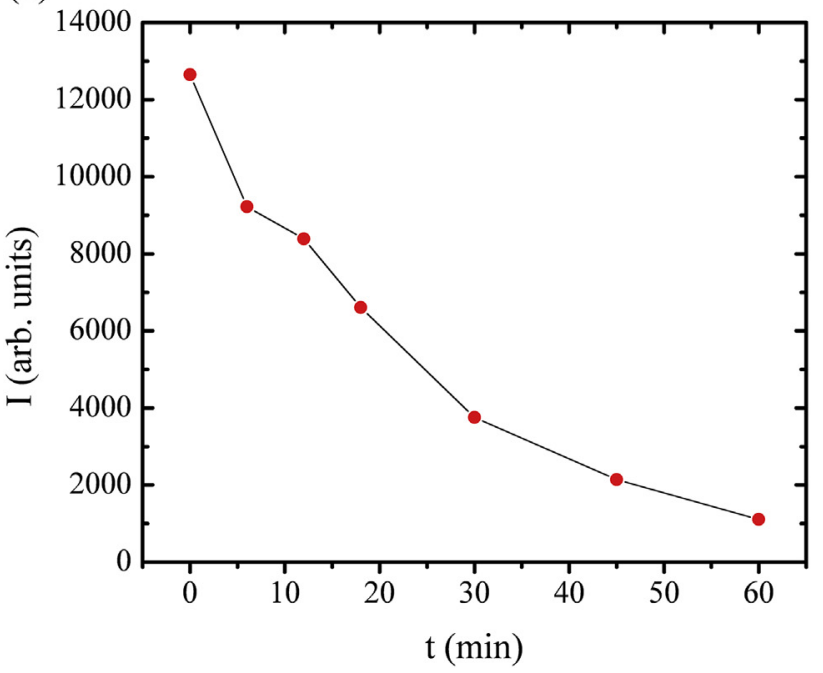

Fig. 4. (a) Dy growth at $130 \mathrm{~K}, \mathrm{E}=142 \mathrm{eV}$ with shorter stepwise depositions of $0,6,12$, $18,30,45$, and $60 \mathrm{~min}$ with flux rate $1 / 30 \mathrm{ML} / \mathrm{min}$. The profiles are along the [1100] direction with the two outside spots the graphene spots. The graphene spots consist of two components (a sharp and a very broad component one similar to the 00 spot discussed earlier). The initial intensity (black) and the final intensity (blue) show the total change on the surface after Dy deposition. (b) An example of how a typical profile is decomposed into a sharp and broad components which were used to estimate the integrated intensities of the graphene spot (this profile is for 12 min deposition). (c)The integrated intensity of the graphene central spike plotted vs deposition time. After 60 min of deposition the integrated graphene intensity drops to less than $8 \%$ its initial value, which shows that graphene is effectively covered by the Dy film. (A colour version of this figure can be viewed online.)

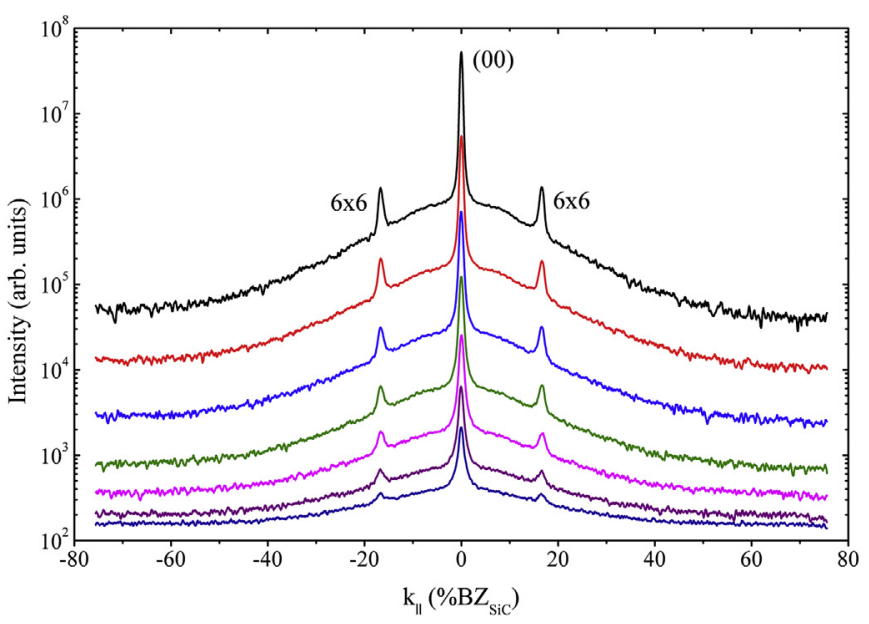

Fig. 5. LEED 1-d line profiles scanned along [1 $\overline{2} 10] \mathrm{E}=142 \mathrm{eV}$ for the stepwise depositions described for Fig. (4). All three components of the 00 spot (the broad background, the central spike and the $6 \times 6$ spots) decrease gradually in intensity. The final $6 \times 6$ intensity (black) shows is less than $5 \%$ its initial value which confirms that graphene is covered by the Dy film. (A colour version of this figure can be viewed online.)

island edge is along $90^{\circ}$ from the graphene lattice direction. This is one of the three equivalent [ 1210$]$ directions consistent with the orientation of the scan of Fig. 2(a) and with the binding site of Dy being the $\mathrm{H}$ site within the graphene unit cell [21].

Fig. 3 shows the result after deposition of $2 \mathrm{ML}$ at three different temperatures over a wide temperature range (650 K, RT, $130 \mathrm{~K}$ ) along the [12 10] direction with all profiles measured at $130 \mathrm{~K}$. No spot is discerned after deposition at $130 \mathrm{~K}$ (bottom). After deposition at RT Dy spots with much lower intensity than the strong spot discussed in Fig. 2(a) are observed (middle). These results show that crystalline Dy islands grow at room temperature or above. For deposition at lower temperatures the film is amorphous. The presence of a strong 00 spot indicates that although the film is amorphous and there is no lateral long range order, it is of uniform thickness and of minimal roughness.

The integrated intensity of the Dy spot is a measure of the crystalline fraction of the film. Since the same total amount of $2 \mathrm{ML}$ Dy is deposited in the experiment of Fig. 3, this shows the integrated intensity of the Dy spot (and therefore crystallinity) increases gradually with growth temperature. In addition monitoring the temperature dependence of the $6 \times 6$ spot is a way to follow how Dy is distributed laterally, since the $6 \times 6$ spot measures the uncovered area of graphene. The drop of the $6 \times 6$ intensity is highest at $130 \mathrm{~K}$ suggesting that the Dy film is thinner and covering larger areas. Controlled annealing of the $130 \mathrm{~K}$ film and monitoring the corresponding changes to the Dy and $6 \times 6$ spots may be used to induce crystallization while maintaining lower film thickness.

Figs. 4 and 5 depict results from targeted experiments at $130 \mathrm{~K}$ with shorter stepwise depositions of $0,6,12,18,30,45$, and $60 \mathrm{~min}$ for a total deposited amount of $2 \mathrm{ML}$ at a flux rate of $1 / 30 \mathrm{ML} / \mathrm{min}$. The evolution of the profiles are gradual and all spots along both scanned directions decrease in intensity. Fig. 4(a) shows the profiles along [1 $1 \overline{1} 00]$. The initial intensity profile (black) and the final intensity profile (blue) reflect the total change on the surface. Initially the graphene spots consist of two components (as for the 00 spot discussed earlier): the central spike and the broad component. With successive Dy depositions the broad component decreases as the profile wings become flatter and the central spike drops in intensity by an order of magnitude. Fig. 4(b) depicts a typical fit of the profiles which is used to extract the different components of 

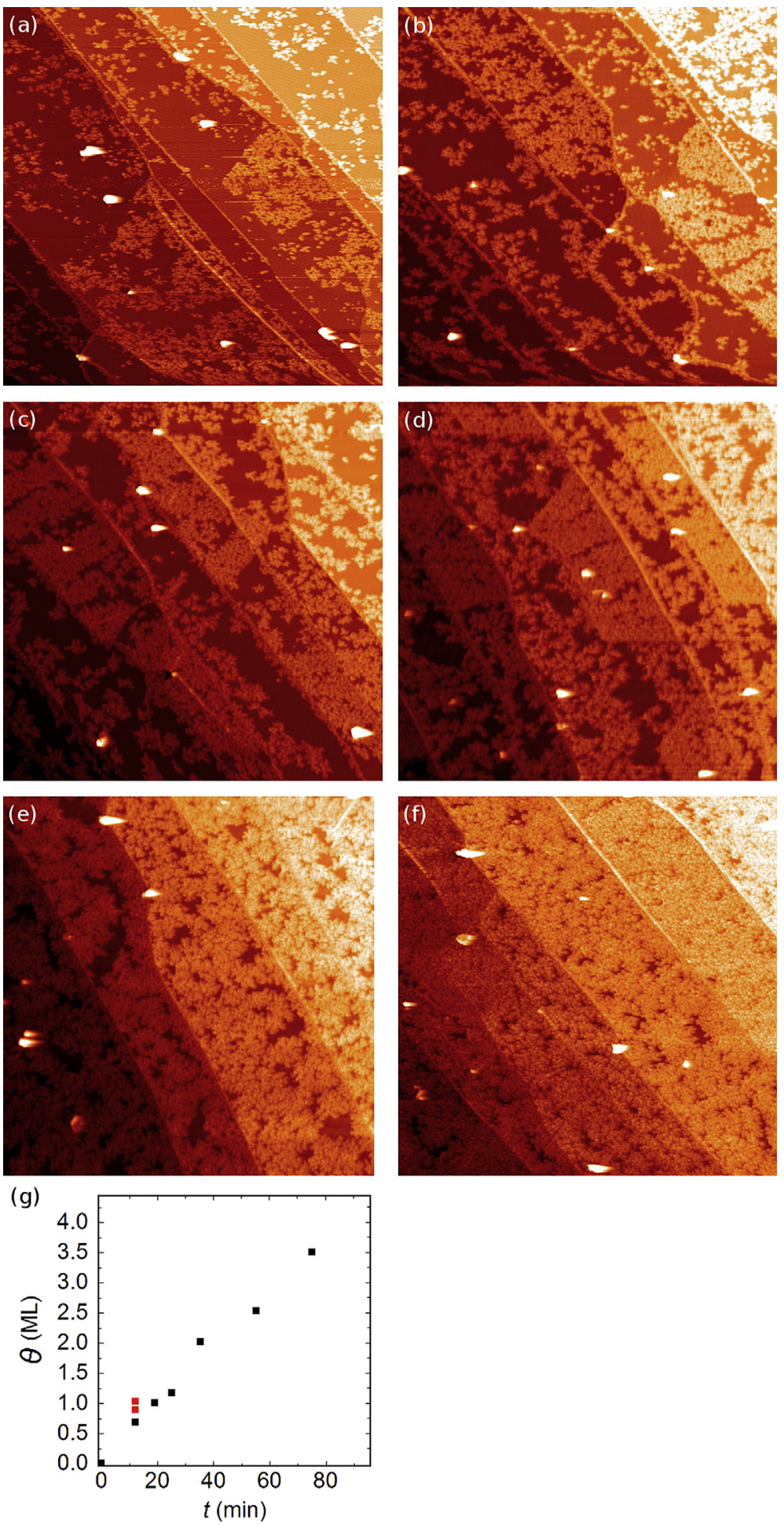

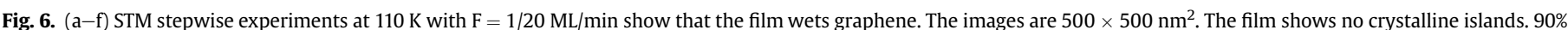

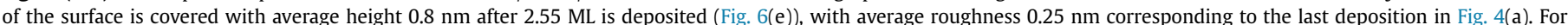

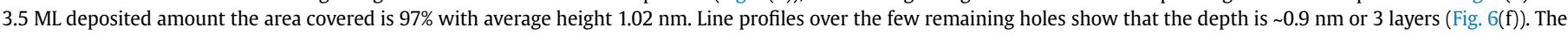

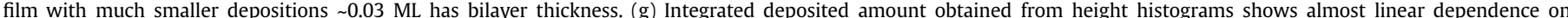

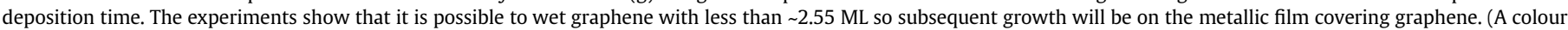
version of this figure can be viewed online.)

each spot (i.e. central spike and broad components for the graphene spots and central spike, broad and $6 \times 6$ for the 00 spot discussed previously in connection to Fig. 1. Similar changes are seen for the 00 spot in Fig. 4(a) along [1100] (scanned $30^{\circ}$ from the direction in
Fig. 1 with the central spike reduced, after Dy deposition, to less than $6 \%$ its initial value. The integrated intensity of the graphene central spike from Fig. 4(a) is plotted in Fig. 4(c) and after 60 min of deposition it becomes less than $8 \%$ of its initial value. This shows 


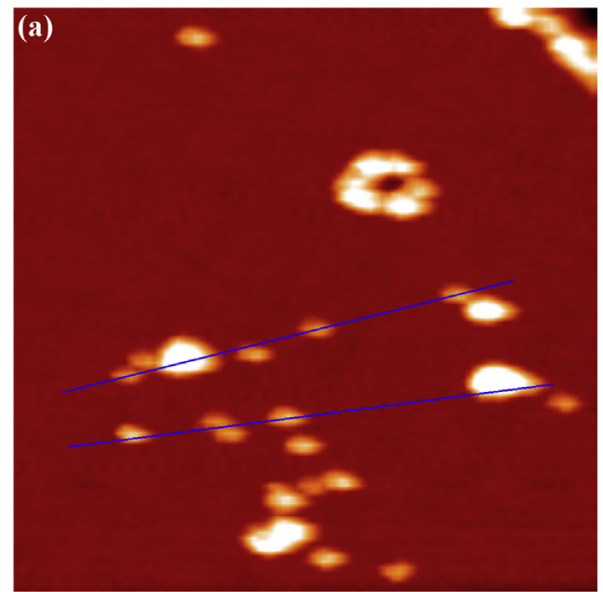

(b)

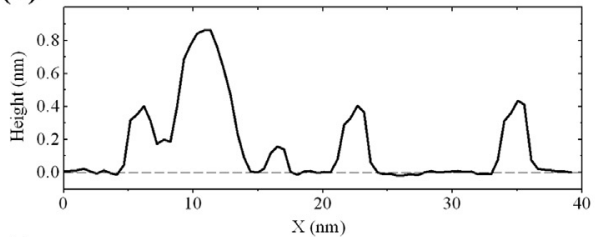

(c)

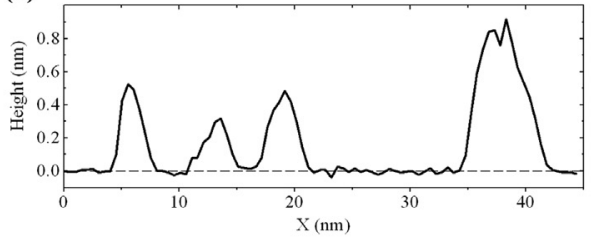

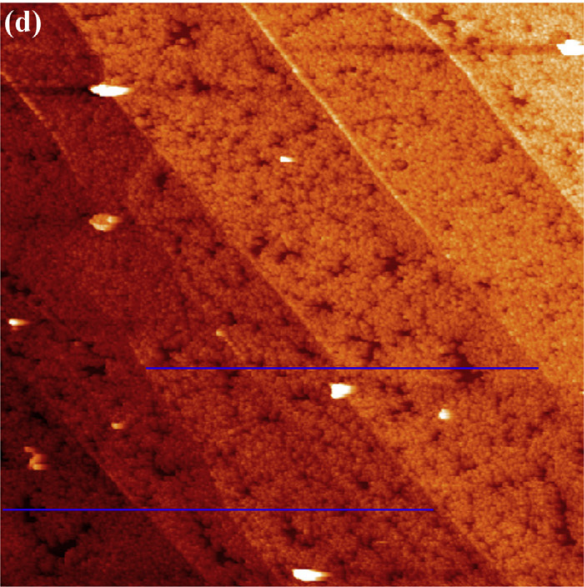

(e)

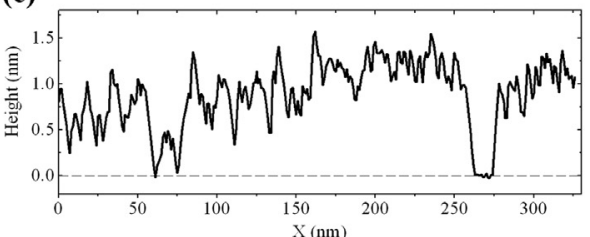

(f)

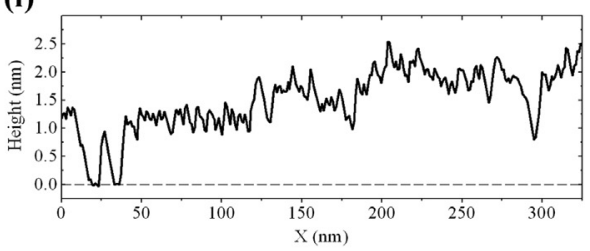

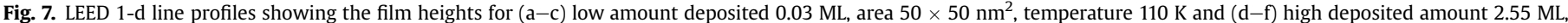
area $500 \times 500 \mathrm{~nm}^{2}$, temperature $300 \mathrm{~K}$. (A colour version of this figure can be viewed online.)

that the graphene is effectively covered by an amorphous Dy film (initially with bilayer thickness as also seen in the STM scans of Fig. 6 to be discussed later).

These conclusions are further supported by the profiles of Fig. 5 scanned along [12 10]. All three components of the 00 spot (the broad background, the central spike and the $6 \times 6$ spots) decrease gradually in intensity. The final $6 \times 6$ intensity (blue) is very close to zero, confirming that graphene is almost fully covered by Dy.

The conclusion that graphene is effectively wetted by Dy at $130 \mathrm{~K}$ is confirmed from the STM experiments at $110 \mathrm{~K}$ shown in Fig. 6. The Dy deposition was carried out with $F=1 / 20 \mathrm{ML} / \mathrm{min}$. The series of STM images in Fig. 6 show that the deposited film extends gradually and covers $90 \%$ of the surface with average height $0.8 \mathrm{~nm}$ after $2.55 \mathrm{ML}$ is deposited. The film shows no islands and its growth front has an average roughness of $0.26 \mathrm{~nm}$. For 3.5 ML deposited the area covered is $97 \%$ with an average height $1.02 \mathrm{~nm}$ as shown in Fig. 6(f). Line profiles over the few remaining holes in Fig. 6(f) show that the depth is $\sim 0.9 \mathrm{~nm}$ or 3 layers. It is also interesting for the lowest Dy amount of 0.7 ML in Fig. 6 with an area of 35\% covered that the average height is $0.56 \mathrm{~nm}$ (Fig. 4) reflecting that already very early the film grows with bilayer thickness. In comparing Figs. 4 and 6 the final profile in Fig. 4(a) is closer to Fig. 6(e) and the slight difference in Dy amount to cover graphene is due to the lower flux rate in the SPA-LEED experiment.

In other experiments when only $\sim 0.03 \mathrm{ML}$ is deposited the average height is $0.4 \mathrm{~nm}$ showing that the initial few-atom islands are predominantly bilayer, too. This is surprising because one expects both diffusion along the surface and upward transfer of atoms to be suppressed at such low temperature, so the very initial amorphous layer should have been 1-layer high. It is not clear what is the driving force for transferring material to the second layer instead of material being added to the island sides to extend them laterally. Fig. 6(g) shows the integrated deposited amount obtained from height histograms which record the probability of a certain height to be present. The curve is almost linear with deposition time which indicates that the sticking coefficient, well below room temperature, is high initially on graphene and on amorphous Dy as subsequent layers grow.

Fig. 7 shows $1-d$ scans at low $(a-c)$ and high coverage $(d-f)$ indicating that the film height starts from bilayer thickness. At low temperatures the step edge barrier of the amorphous film is asymmetric,i.e., transport from higher to lower layers is high while transfer in the opposite direction is suppressed. One reason for the asymmetry is most likely the cohesive energy of an amorphous film is much less than the cohesive energy of a crystalline film.

Fig. 8 shows how semi-crystalline islands start forming at room temperature. This is seen from the islands having compact shapes (but still with incomplete top layers); and height histograms that shows peaks corresponding to the interlayer spacing $0.28 \mathrm{~nm}$ of crystalline Dy. These results imply that controlled experiments as a function of temperature and annealing time would crystallize the amorphous film (also needed for ref. [17]) without affecting its height uniformity.

\section{Discussion}

The experiments have shown that the growth of Dy depends dramatically on temperature with wetting of graphene possible at 

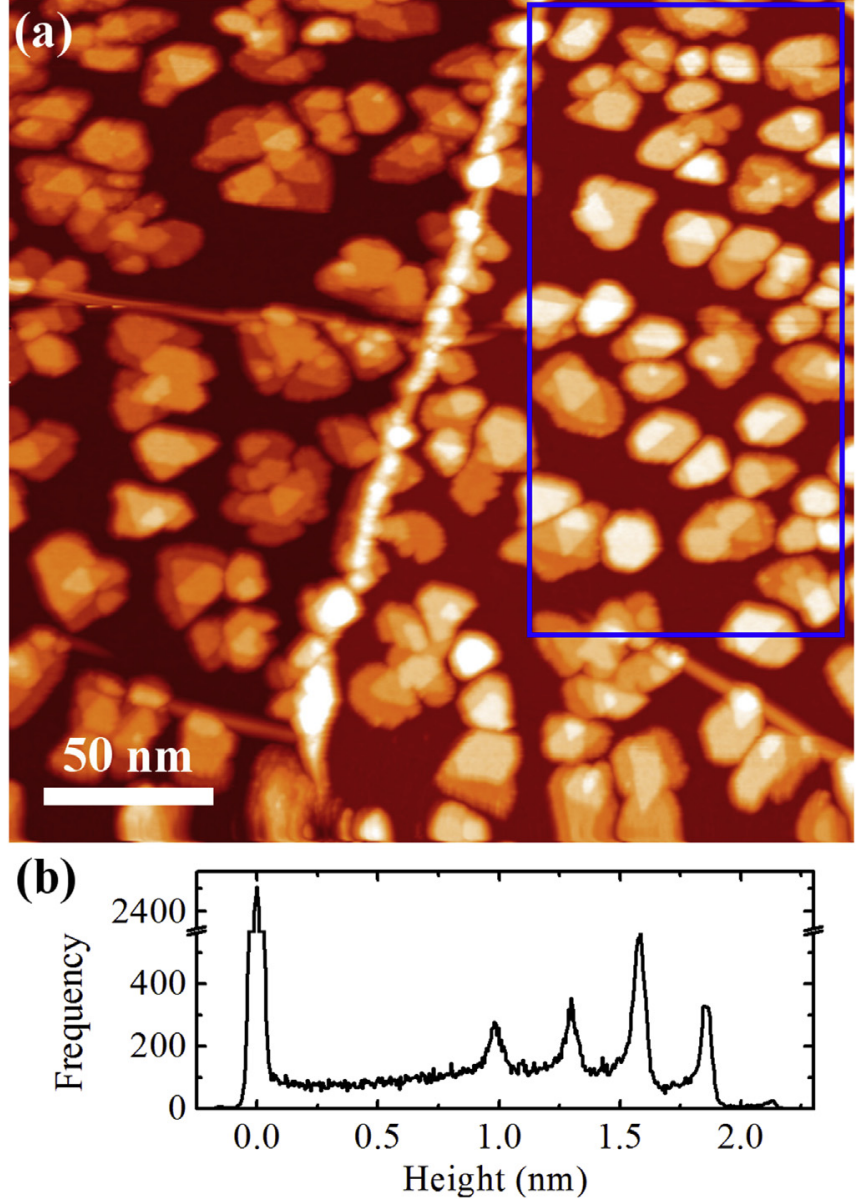

Fig. 8. STM images after growth of $2.66 \mathrm{ML}$ at $300 \mathrm{~K}$ showing compact islands with incomplete top layers. The peaks in the height histogram correspond to $0.98 \mathrm{~nm}$, $1.3 \mathrm{~nm}, 1.58 \mathrm{~nm}, 1.86 \mathrm{~nm}$, and $2.13 \mathrm{~nm}$ height, differing by $0.28 \mathrm{~nm}$, i.e., the Dy interlayer spacing. This indicates the formation of mostly crystalline islands. (A colour version of this figure can be viewed online.)

$\sim 110 \mathrm{~K}$ and with $\sim 2.5 \mathrm{ML}$ of Dy. The SPA-LEED diffraction patterns and 1-d scans monitor several complementary spots that measure the amount of exposed graphene areas. The gradual decay of these spots with Dy shows that graphene is covered and 3D growth is avoided.

These results show that manipulation of the kinetics can bypass the large difference between Dy-Dy and Dy-carbon bonding which favors 3D growth close to equilibrium. Kinetic manipulation was used to change $3 \mathrm{D}$ to $2 \mathrm{D}$ growth by growing films at high flux rate, at low temperatures or in pre-seeding experiments where a small amount of a strongly bound metal can induce layer-by-layer growth for a weakly bound metal [16l. These effects were found either to reduce the step edge barrier at the edge of a growing island so interlayer transfer is enhanced; or to reduce adatom mobility so diffusion to pre-existing islands and transfer to higher layers is suppressed. The suppression of upward mass transfer seems to be the reason for the low temperature Dy film height uniformity grown on graphene. After the graphene is fully covered, layer-bylayer becomes more likely because the growth of the next layers will be controlled by the barriers of the metal on metal system. It is still intriguing for Dy on graphene why the first nucleated islands observed at $\sim 110 \mathrm{~K}$ are not single layer but bilayer. At the onset of nucleation, it is energetically favorable to transfer material on top of the small first layer islands, which have amorphous structures, instead of extending them laterally.
Since the Dy film at $110 \mathrm{~K}$ is amorphous but of small roughness ( $~ 0.25 \mathrm{~nm}$ which is less than one atomic layer), the lack of long range order implies that its electronic properties and conductivity will be worse than the corresponding properties of a crystalline Dy film of similar thickness. Annealing experiments will promote crystallization but they need to be finely tuned, so crystallization happens without transferring Dy atoms to higher layers.

2D growth on graphene (grown on $\operatorname{Ir}(111)$ ) was also demonstrated in Co films using LPD with film thickness in the range of $0.5-1 \mathrm{~nm}$ [17]. The 2D growth was attributed to an effective higher flux (i.e., $10^{6}$ times the typical rates $\sim 1 \mathrm{ML} / \mathrm{min}$ used in molecular beam epitaxy) that results in high density of smaller islands which promote interlayer diffusion. The magnetic properties of the Co film were studied with DFT and ex-situ with SMOKE. Because the Co film most likely is also amorphous (as STM images show no periodicity and a texture similar to the Dy films) the magnetic properties are not optimal. The magnetization of the film must be below the magnetization expected for crystalline Co films.

Besides thermodynamic factors that favor 3D growth for metals on graphene, there are other energetic reasons favoring 3D growth, as seen in experiments aimed to minimize the contact resistance on graphene treated with $\mathrm{O}_{2}$ plasma [1]. Various metals ( $\mathrm{Al}, \mathrm{Ti}, \mathrm{Cu}, \mathrm{Ni}$, $\mathrm{Pt}$ ) were first deposited prior to depositing $\mathrm{Au}$ (the main metal for the contacts) to test whether larger work function differences lead to better Ohmic contacts. No such correlation was found and all metals were found to grow 3D. Although large work function differences were expected to be beneficial because of the increasing number of carriers, they can lead to island charging. This should be an effect observed in substrates which lack free, highly mobile electrons to screen the transfer charge at the interface [22]. Graphene is such a substrate because of the low density of states; even for graphene on $\mathrm{SiC}$, used in the current experiments with the Fermi level $0.42 \mathrm{eV}$ above the Dirac cone [23], the carrier density is $\sim 2$ orders of magnitude below the metal carrier density. There is no significant screening of the dipoles formed at the metal graphene interface, so Coulomb repulsive interactions within or between these charged metal islands cost energy and can have a large effect in the grown morphology. Since the charge transfer is determined by the island footprint (and increases with work function mismatch $\Delta \varphi$ ) charging favors 3D growth and islands with higher height/ footprint ratios. This factor can be applicable both at equilibrium and non-equilibrium growth conditions; for crystalline or amorphous islands [20].

Effects related to the interplay of the three factors discussed so far (the ratio of metal-carbon to metal cohesive energy $E_{\mathrm{a}} / E_{\mathrm{c}}$, the work function difference $\Delta \varphi$ and Coulomb repulsion) have been seen already in different systems and over a wide range of growth conditions (of temperature and coverage). For Eu on graphene on $\operatorname{Ir}(111)$ at $35 \mathrm{~K}$ the island nucleation was studied as a function of coverage $\theta$ [24]. Contrary to classical theory of nucleation, where steady state is attained with constant island density, Coulomb repulsion keeps the diffusing adatoms away from dimers and it increases the dimer density at lower $\theta$. At some critical $\theta_{c}$ the gain in cohesive energy and/or the reduction of the electron kinetic energy (if the initial Fermi levels across the interface are mismatched) favor bigger clusters. Coulomb repulsion effectively amounts to negative cohesive energy for very small island sizes, thus maintaining initially dimers as the stable species. In a different growth study of Au deposition on multilayer graphene (MLG) (exfoilated graphene on $\mathrm{SiO}_{2}$ ) it was found that the nucleated island density was a strong function of MLG thickness $m$ [22]. This again was accounted for by two competing effects: the cohesive energy which favors islands with larger areas and the Coulomb repulsion, which favor islands with smaller footprint (and thus larger height). As $\mathrm{m}$ increases screening becomes more important, and reduces effects 
related to the Coulomb repulsion. For fixed $\theta$, nucleation as a function of $m$ shows that islands with larger areas are favored, thus the nucleated island density decreases with MLG graphene thickness. All types of energy terms mentioned (metal adsorption energy, metal cohesive energy, charge transfer to match Fermi levels, Coulomb repulsion) are relevant both to crystalline and/or amorphous islands, but the exact values for each case should be worked out for any specific metal.

Since there are other factors that favor 3D growth of metals on graphene besides thermodynamics (the ratio $E_{\mathrm{a}} / E_{\mathrm{c}}$ ), it is not clear how to find generic ways to manipulate $3 \mathrm{D}$ to $2 \mathrm{D}$ growth. A scheme was suggested to enhance the bonding energy of a weakly interacting metal M1 by using a metal-graphene-metal (M2-G-M1) sandwich geometry, with a strongly bound metal M2 grown below graphene [25]. As shown experimentally the large charge transfer at the M2-G interface and the induced dipoles can increase the binding energy of $\mathrm{M} 1$ on graphene, enhance the ratio of $E_{\mathrm{a}} / E_{\mathrm{c}}$ and can result in 2D morphology. Reduced contact resistance was accomplished for the sandwiched structures for Ti-G-Pd. Many other possibilities can be realized with a much richer library of combinations M2-G-M1 to be tested.

Developing a good understanding of the factors controlling the grown metal morphology on graphene is also important for metal growth on 2D layered materials like $\mathrm{MoS}_{2}$ [26]. The material is also of intense interest for future microelectronic applications, since it has high carrier mobility. It is essential to achieve uniform wetting of metals on $\mathrm{MoS}_{2}$. 3D growth was also found after deposition of several metals $(\mathrm{Ag}, \mathrm{Au}, \mathrm{Pd})$ again because of the low ratio $E_{\mathrm{a}} / E_{\mathrm{c}}$ ratio. Both the kinetic approaches discussed, exploiting the competition between different energetic factors that promote 3D growth and interface engineering in sandwich geometries in graphene, are very relevant for metal growth on $\mathrm{MoS}_{2}$ and other transition metal dichalcogenides.

\section{Conclusions}

The growth of Dy on graphene was studied with complementary techniques of SPA-LEED and STM to search for ways to grow 2D film morphologies on graphene. Identifying such a growth mode is essential because most metals studied so far show that growth is $3 \mathrm{D}$, either because of the relatively small metal-carbon bond or because poor screening of charges transferred at the metal graphene interface favor islands with smaller areas. A quantitative spot profile analysis of LEED spots during stepwise deposition experiments well below room temperature $(130 \mathrm{~K})$ show that graphene is covered by the film with the minimum thickness of a Dy bilayer. These findings were confirmed in corresponding STM experiments under similar conditions showing that graphene is essentially covered by the film with 2.5 ML Dy deposited. However, the film is amorphous but it is possible to crystallize it while limiting island height, since as discussed in Fig. 3 and ref. [21] long range order develops gradually with increasing temperature. The significance of these results is broader for metal growth on graphene. Similar welltuned kinetic pathways can be used, to overcome the thermodynamic bottleneck that limits metal growth to be 3D for other metals of interest [27].

\section{Acknowledgments}

This work was supported by the U.S. Department of Energy (DOE), Office of Science, Basic Energy Sciences, Materials Science and Engineering Division. The research was performed at Ames Laboratory, which is operated for the U.S. DOE by Iowa State University under contract \# DE-AC02-07CH11358. H.H. was sponsored by a postdoctoral fellowship of the Leopoldina Fellowship Program LPDS 2013-04 of the German National Academy of Sciences.

\section{References}

[1] J.A. Robinson, M. LaBella, K.A. Trumbull, X.J. Weng, R. Cavalero, T. Daniels, Z. Hughes, M. Hollander, M. Fanton, D. Snyder, Contacting graphene, Appl. Phys. Lett. 98 (2011) 053103.

[2] A. Venugopal, L. Colombo, E.M. Vogel, Contact resistance in few and multilayer graphene devices, Appl. Phys. Lett. 96 (2010) 013512.

[3] K. Nagashio, T. Nishimura, K. Kita, A. Toriumi, Metal/graphene contact as a performance killer of ultra-high mobility graphene-Analysis of intrinsic mobility ntact resistance, Tech. Dig. Int. Electron Devices Meet. (2009) $565-5652$

[4] S. Russo, Tr.r. Craciun, M. Yamamoto, A.F. Morpurgo, S. Tarucha, Contact resistance in graphene-based devices, Phys. E 42 (2010) 677.

[5] J.S. Moon, D. Curtis, S. Bui, T. Marshall, D. Wheeler, I. Valles, S. Kim, E. Wang, X. Weng, M. Fanton, Top-gated graphene field-effect transistors using graphene on Si (111) wafers, IEEE Electron Device Lett. 31 (2010) 1193.

[6] J.S. Moon, D. Curtis, S. Bui, M. Hu, D.K. Gaskill, J.L. Tedesco, P. Asbeck G.G. Jernigan, B.L. VanMil, R.L. Myers-Ward, C.R. Eddy, P.M. Campbell, X. Weng, Top-gated epitaxial graphene FETs on Si-face SiC wafers with a peak transconductance of $600 \mathrm{mS} / \mathrm{mm}$, IEEE Electron Device Lett. 31 (2010) 260.

[7] V.M. Karpan, G. Giovannetti, P.A. Khomyakov, M. Talanana, A.A. Starikov, M. Zwierzycki, J. van den Brink, G. Brocks, P.J. Kelly, Graphite and graphene as perfect spin filters, Phys. Rev. Lett. 99 (2007) 176602.

[8] M. Hupalo, E.H. Conrad, M.C. Tringides, Growth mechanism for epitaxial graphene on vicinal $6 \mathrm{H}-\mathrm{SiC}(0001)$ surfaces: a scanning tunneling microscopy study, Phys. Rev. B 80 (2009) 041401.

[9] X. Liu, C.Z. Wang, Y.X. Yao, W.C. Lu, M. Hupalo, M.C. Tringides, K.M. Ho, Metals on graphene: correlation between adatom adsorption behavior and growth morphology, Phys. Chem. Chem. Phys. 14 (2012) 9157.

[10] E.J. Kwolek, et al., Adsorption of Dy on the graphite (0001) surface: nucleation and growth at $300 \mathrm{~K}, \mathrm{~J}$. Chem. Phys. (2016) (in press).

[11] X. Liu, Y. Han, J.W. Evans, A.K. Engstfeld, R.J. Behm, M.C. Tringides, M. Hupalo, H.Q. Lin, Li Huang, K.M. Ho, D. Appy, P.A. Thiel, C.Z. Wang, Growth morphology and properties of metals on graphene, Progr. Surf. Sci. 90 (2015) 397.

[12] S.M. Binz, M. Hupalo, X.J. Liu, C.Z. Wang, W.C. Lu, P.A. Thiel, K.M. Ho E.H. Conrad, M.C. Tringides, High island densities and long range repulsive interactions: Fe on epitaxial graphene, Phys. Rev. Lett. 109 (2012) 026103.

[13] J.W. Evans, P.A. Thiel, M.C. Bartelt, Morphological evolution during epitaxia thin film growth: formation of 2D islands and 3D mounds, Surf. Sci. Rep. 61 (2006) 1.

[14] R. Zan, U. Bangert, Q. Ramasse, K.S. Novoselov, Metal-graphene interaction studied via atomic resolution scanning transmission electron microscopy, Nano Lett. 11 (2011) 1087.

[15] C.J. Shih, Q. H.Wang, S. Lin, K.C. Park, Z. Jin, M.S. Strano, D. Blankschtein, Breakdown in the wetting transparency of graphene, Phys. Rev. Lett. 109 (2012) 176101

[16] M.G. Lagally, Atom motion on surfaces, Phys. Today 46 (1993) 24.

[17] C. Vo-Van, Z. Kassir-Bodon, H. Yang, J. Coraux, J. Vogel, S. Pizzini, P. BayleGuillemaud, M. Chshiev, L. Ranno, V. Guisset, P. David, V. Salvador, O. Fruchart Ultrathin epitaxial cobalt films on graphene for spintronic investigations and applications, New J. Phys. 12 (2010) 103040.

[18] A.M. Clausen, D.M. Paskiewicz, A. Sadeghirad, J. Jakes, D.E. Savage, D.S. Stone, F. Liu, M.G. Lagally, Silicon nanomembranes as a means to evaluate stress evolution in deposited thin films, Extrem. Mech. Lett. 1 (2014) 9.

[19] M.T. Hershberger, et al. (in preparation).

[20] D. McDougall et al. (in preparation).

[21] M.T. Hershberger, M. Hupalo, P.A. Thiel, M.C. Tringides, Growth of fcc(111) Dy multi-height islands on 6H-SiC(0001) graphene, J. Phys. Cond. Mat. 25 (2013) 225005.

[22] Z. Luo, L.A. Somers, Y. Dan, T. Ly, N.J. Kybert, E.J. Mele, A.T.C. Johnson, Sizeselective nanoparticle growth on few-layer graphene films, Nano Lett. 10 (2010) 777

[23] I. Gierz, C. Riedl, U. Starke, C.R. Ast, K. Kern, Atomic hole doping of graphene Nano Lett. 8 (2008) 4603

[24] D.F. Forster, T.O. Wehling, S. Schumacher, A. Rosch, T. Michely, Phase coexistence of clusters and islands: europium on graphene, New J. Phys 14 (2012) 023022

[25] C. Gong, D. Hinojos, W. Wang, N. Nijem, B. Shan, R.M. Wallace, K. Cho, Y.J. Chabal, Metal-graphene-metal sandwich contacts for enhanced interface bonding and work function control, ACS Nano 6 (2012) 5381.

[26] C. Gong, C. Huang, J. Miller, L. Cheng, Y. Hao, D. Cobden, J. Kim, R.S. Ruoff, Robert M. Wallace, K. Cho, X. Xu, Y.J. Chabal, Metal contacts on physical vapor deposited monolayer MoS2, ACS Nano 7 (2013) 11350.

[27] S. Sarkar, M.L. Moser, X. Tian, X. Zhang, Y. Fadel Al-Hadeethi, C. Haddon, Metals on graphene and carbon nanotube surfaces: from mobile atoms to atomtronics to bulk metals to clusters and catalysts, Chem. Mater 26 (2014) 184. 